\title{
A 7 year follow-up of children and adolescents with obsessive-compulsive disorder. An analysis of predictive factors in a clinical prospective study
}

\author{
Judith Becker Nissen, MD, PhD \\ Kathrine Hesselberg Nikolajsen, MD \\ Per Hove Thomsen, Professor, MD
}

The University Hospital of Aarhus, Children and Adolescent Psychiatric Centre, Risskov

DENMARK

\begin{abstract}
Background and Objectives: Obsessive compulsive disorder (OCD) is a frequent psychiatric disorder. Despite its significant influence on personal development, little is known about its long-term course in children and adolescents. The aim was to follow children and adolescents with OCD for 7 years and to compare patients gaining remission with patients experiencing symptoms.

Methods: The study was a prospective follow-up study in which the patients were interviewed 7 years after their initial contact with the healthcare system. The patients had been described at baseline.

Results: Among the 95 participants, 53 participants described symptoms at follow-up and 44 participants described remission. More patients with persistent symptoms described an anxious personality trait. The schizoid/compulsive personality traits were represented at a higher rate in the group with persistent symptoms than in the group with remission symptoms. Important predictive factors included predisposition to OCD, the occurrence of comorbid disorders and older referral age. The occurrence of magic obsessions and repetitive compulsions increased the risk of OC symptoms at follow-up. The group "late responders" differed from the non-responder group by predisposition to depression.

Conclusions: The present clinical study is one of the longest follow-up studies conducted in children and adolescents with OCD. In addition to confirming several previous findings, the study added new knowledge about the importance of phenotypic presentation, personality traits and quality of life. Furthermore, the late responder group has been described in relation to the remission group and to the group with persistent symptoms.
\end{abstract}




\section{Introduction}

Obsessive compulsive disorder (OCD) is a frequent psychiatric disorder characterised by obsessions, compulsions and an often intense and disabling fear. Among children and adolescents, $1-2 \%$ meet the criteria for OCD. Most of the affected persons describe an early age of onset, and the disorder often severely impacts their education and personal development. Despite this negative influence, surprisingly little is known about the long-term course and prognosis of OCD in children and adolescents.

The disorder is treated with cognitive behavioural therapy (CBT) and antidepressants. Several studies have investigated the shortterm outcome of treatment with CBT and medication. Most of these studies show a significant effect of treatment with CBT, medication and combined treatment as compared with placebo ${ }^{1-3}$.

Thomsen et al. ${ }^{4}$ examined children and adolescents with OCD 1.5 to 5 years after admission to the hospital. Approximately half of the patients still had the diagnosis of OCD, which ran an episodic course in $1 / 3$ and a more chronic course in $2 / 3$ of these patients. A comparable result was seen in a study by Leonard HL et al. ${ }^{5}$ in which children and adolescents were re-evaluated 2-7 years after treatment with an antidepressant. On followup, $43 \%$ met the diagnostic criteria for OCD and $6 \%$ were in remission. The majority continued taking medication. Negative predictive factors included severe OCD symptoms after initiation of treatment, a lifetime history of a tic disorder and the presence of parental axis I psychiatric diagnoses. In a metaanalysis and qualitative review from 2004, the longterm outcome of child and adolescent-onset OCD was examined. The follow-up intervals ranged between 1 and 15.6 years. The mean persistence rate was $41 \%$ for full OCD and $60 \%$ for full or sub-threshold OCD. Negative predictive factors included an early age of onset. In-patient status predicted greater symptom persistence ${ }^{6}$. Furthermore, comorbid psychiatric illness and poor initial treatment response were negative prognostic factors. The authors stressed that the long-term persistence of OCD with onset in children and adolescents may be lower than previously thought ${ }^{6,7}$. Micali $\mathrm{N}$ et al. examined the persistence of OCD after 9 years and showed a persistence rate of $41 \%$. About $50 \%$ of the patients continued receiving treatment. The main predictor of OCD was the duration of the illness ${ }^{8}$. The authors concluded that paediatric OCD may be a chronic condition.

Overall, studies on long-term follow-up of OCD in children and adolescent have shown that a substantial portion of patients experience remission at follow-up. As such, the prognosis in children and adolescents seems to be better than in adults ${ }^{7}$. However, most studies show that $40-60 \%$ may continue having symptoms of varying degrees of severity, which indicates that OCD may be a chronic condition in some patients.

The aim of the present clinical prospective study was to follow a large group of children and adolescents with OCD for 7 years and to compare the group of patients gaining remission with the group of patients experiencing continuous OC symptoms. The duration of the follow-up period is one of the longest in children and adolescents with OCD. Furthermore, we wanted to investigate possible predictors of outcome. We hypothesised that a substantial number of patients would experience OC symptoms after 7 years and that a poor prognosis would be associated with early age of onset, severe OCD at baseline and the occurrence of comorbidity. Furthermore, we expected that the phenotypic expression of ordering/symmetry and hoarding would have a 
negative influence on the long-term course of the disorder. Finally, we hypothesised that a number of patients would experience a late reduction in OC symptoms.

\section{Methods}

The study was a prospective follow-up study of a cohort of 134 subjects. Most of the children and adolescents had been described at baseline ${ }^{9}$. The detailed sample characteristics at baseline are described more thoroughly elsewhere and a summary is presented in table $1^{9}$. In the retrospective study, the study population included consecutive children and adolescents fulfilling the inclusion criterias which were a primary or secondary OCD diagnosis according to the International Classification of Diseases, $10^{\text {th }}$ edition (ICD-10) with a measured total CYBOCS score. Exclusion diagnosis included psychosis (F20-29), pervasive developmental disorder (F84-84.9) and mental retardation (F70-79). In the retrospective study, all patients had been diagnosed by psychiatric evaluation conducted by at least two experienced clinicians, either a psychiatrist or a psychologist, using systematic clinical interviews of both parents and their child designed to elicit ICD-10 diagnoses. The parents and the child had been asked about all the OCD symptoms in the ICD-10 and in the Children's Yale-Brown Obsessive-Compulsive Scale (CY-BOCS), and symptom checklists had been completed at baseline. In addition to the severity scale and the symptom checklist, the degree of insight had been evaluated according to the general assessment part in the CY-BOCS. The clinical diagnosis was confirmed at a clinical conference attended by the involved psychiatrist or psychologist and a consultant psychiatrist.
In the present follow-up study, the parents or the patients were contacted by telephone 7 years after their initial contact to the clinic. The interview was conducted by a child and adolescent psychiatrist with specialised training in OCD (JBN). The patients who agreed to participate in the study were interviewed using an interview guide addressing the experience of continuous OC symptoms after discharge from the hospital and at the time of follow-up. If the patients still had symptoms, the symptoms were evaluated using the CYBOCS score $^{10}$. The score ranged from 0-40 dividing the severity into four groups: subclinical OCD (1-9), mild OCD (10-18), moderate OCD (19-29) and severe OCD (30-40) $)^{11}$. Thus, description of any OC symptoms was computed as being symptomatic at either discharge and/or at follow-up.

Probands denying OC symptoms at follow-up were considered remitters. Furthermore, the patients were interviewed about the occurrence of other psychiatric symptoms (tic, depressive or anxiety symptoms, eating, or educational problems, and psychotic symptoms) and lifetime medication. Finally, they were invited to answer questions concerning the occurrence of psychiatric symptoms in family members (symptoms present/not present), quality of life and characteristic personality traits. Questions concerning the psychiatric symptoms in the family were dichotomised and included OC, tic, anxiety, depressive, and psychotic symptoms. Quality of life was rated on a Likert scale (0-5) rating quality of life in periods with and without OCD. Questions included "Have I been happy?", "Have I felt relaxed and calm?", "Have I felt active and energetic?", "Do I get refreshed after sleep?", "Has my daily life been filled with things that I find interesting". The maximum score was 25 . Concerning the personality traits, the patients were presented with various statements which they were 
asked to rate on a Likert scale (not true at all -absolutely correct). The statements addressed features from the diagnostic categories. If the patients did not want to fill in the questionnaire, they were asked a few questions concerning the continuance of OC symptoms.

Additional data on all potential patients was collected from the Danish National Psychiatric Register. The Danish National Psychiatric Register has been collecting data of psychiatric admissions since 1969 and on psychiatric admissions, ambulant contacts and emergency visits since 1995 . The register describes diagnoses based on the ICD-8 (until 1994) and the ICD-10 (from 1995).

\section{Data analysis}

Data were analysed using the STATA version 8 software. For the descriptive analysis, frequencies, percentages, means and standard deviations were computed. Group differences for categorical data with few categories were tested with the Fisher's exact test. For continuous or ordered categorical data with many categories, we used the t-tests with unequal variances, which is a robust method of analysis to capture variance heterogeneity and departures of normality ${ }^{12} . p<0.05$ was considered significant. The effects of age at referral, OCD severity (total CYBOCS score) at baseline, sex, number of lifetime Axis I disorders and the phenotype of the OC symptoms on the continuation of OC symptoms were examined in groups using binary regression analyses for relative risks. A p-value of less than 0.05 was considered statistically significant.

We included only three or four risk factors in the regression analysis to maintain the rule of thumb of 10 events per variable in a binary regression analysis ${ }^{14}$. We applied four different regression models: A. Age of referral > 10 years, gender; B. Comorbidity at baseline, tic, depressive symptoms, eating disorder symptoms, hyperkinetic disorder, or conduct disorder; C. Family predisposition to OCD diagnosed, depressive symptoms, hyperkinetic symptoms; D. CYBOCS at baseline, late responsive group, $>10$ years, mean severity level of OC symptoms.

The study was approved by the National Board of Health and the Danish Data Protection Agency (J.nr. 2012-41-0764).

\section{Results}

\section{Demographic data}

Among a total of 134 patients contacted by telephone, $95(71 \%)$ participated in the interview. One person was unable to define and describe potential symptoms. We were unable to locate 30 people, and another eight declined the invitation to participate. The group that did not participate was comparable with the participating group in terms of age at follow-up, gender, CYBOCS score at inclusion, the occurrence of comorbidity, and treatment at baseline (table 1). More females than males (53/95) participated in the interview. However, this gender distribution was comparable to that of the total group. At baseline, 112 (84\%) had an OCD diagnosis as their primary diagnosis. At the time of follow-up, the mean age of the participating group was 20.9 +/- 3.3 (13-28) years (Table 1).

\section{Description of the follow-up group (table 2)}

Among the cohort participating in the study, a total of $71(75 \%)$ reported OC symptoms at discharge from the hospital. Fiftythree patients $(56 \%)$ reported OC symptoms at follow-up (51 patients experienced symp- 
Table 1

Demographic characteristics of the cohort with Obsessive Compulsive Disorder.

\begin{tabular}{lllll} 
& $\begin{array}{l}\text { Original study } \\
\text { group }\end{array}$ & $\begin{array}{l}\text { Study group in the } \\
\text { follow-up study }\end{array}$ & $\begin{array}{l}\text { Group participating } \\
\text { in the follow-up } \\
\text { study }\end{array}$ & $\begin{array}{l}\text { Group not } \\
\text { participating in the } \\
\text { follow-up study }\end{array}$ \\
\hline $\begin{array}{l}\text { Number of } \\
\text { participants }\end{array}$ & 122 & 134 & 95 & 39 \\
\hline $\begin{array}{l}\text { Gender } \\
\begin{array}{l}\text { Males } \\
\text { Females }\end{array}\end{array}$ & $54(44.3 \%)$ & $59(44.0 \%)$ & $42(44,2 \%)$ & $17(43.6 \%)$ \\
\hline $\begin{array}{l}\text { Mean age at referral } \\
\text { Mean age }\end{array}$ & $13.9+/-3.6 \mathrm{yr}$ & $13.9+/-3.7 \mathrm{yr}$ & $13.9+/-3.4 \mathrm{yr}$ & $14.0+/-4.5 \mathrm{yr}$ \\
\begin{tabular}{l} 
at follow-up \\
\hline $\begin{array}{l}\text { OCD at baseline } \\
20.0+/-7.6\end{array}$
\end{tabular} & $20.8+/-3.6 \mathrm{yr}$ & $20.9+/-3.3 \mathrm{yr}$ & $20.6+/-4.2 \mathrm{yr}$ \\
\hline $\begin{array}{l}\text { Comorbidity } \\
69(56.6 \%)\end{array}$ & $85(63.4 \%)$ & $62(65.3 \%)$ & $23(59.0 \%)$ \\
\hline $\begin{array}{l}\text { Predisposition } \\
\text { to OCD }\end{array}$ & $46(34.3 \%)$ & $29(30.5 \%)$ & $17(43.6 \%)$ \\
\hline $\begin{array}{l}\text { Predisposition to } \\
\text { other psychiatric illness }\end{array}$ & $84(62.7 \%)$ & $61(64.2 \%)$ & $23(59.0 \%)$ \\
\hline $\begin{array}{l}\text { Treatment for OCD } \\
\text { at baseline }\end{array}$ & $102(76.1 \%)$ & $73(76.8 \%)$ & $29(74.4 \%)$ \\
\hline $\begin{array}{l}\text { Medication for OCD } \\
\text { at baseline }\end{array}$ & $63(47.0 \%)$ & $46(48.4 \%)$ & $17(43.6 \%)$ \\
\hline
\end{tabular}

Table 2

The distribution of responders and non-responders.

\begin{tabular}{llll} 
& Continued OCD & Yes & No \\
\hline OCD after discharge & & \\
\hline Yes & 51 & 20 (late response) \\
\hline No & 2 (relapse) & 22 (remission) \\
\hline Total & 53 (Non-responders) & 44 (Responders) \\
\hline
\end{tabular}

toms both at discharge and at follow-up; and two patients experienced a relapse, that is they showed no symptoms at discharge, but at follow-up they described OC symptoms) and 20 patients $(21 \%)$ reported OC symptoms after discharge from the hospital, but no symptoms at follow-up (these patients were defined as late responders). A total of 22 pa- tients $(23 \%)$ described no symptoms either after discharge from the hospital or at followup (table 2). At follow-up, 3/53 rated their OC symptoms as severe, $17 / 53$ rated their symptoms as moderate and 20/53 rated their symptoms as mild. Among patients, 19.6\% described a worsening of their symptoms compared with baseline, $37.0 \%$ describe their 
symptoms as unchanged and $43.5 \%$ had experienced recovery. There was no difference between the group with OC symptoms $(\mathrm{N}=$ 53) and the group without symptoms at follow-up $(\mathrm{N}=42)$ in terms of the percentage ever having taken antidepressants (Table 3). However, at follow-up a higher proportion of patients with symptoms continued having medication. A comparable number of patients had other medication in addition to an antidepressant. More patients with symptoms than patients without symptoms at follow-up had used neuroleptic medication (data not shown).

Patients with symptoms at follow-up were also compared with patients with no symptoms at follow-up in terms of their comorbidity, and the two groups had similar levels of comorbidity (Table 3). However, the fraction reporting depressive symptoms ( $p=$ $0.003)$ and eating problems $(p=0.038)$ was significantly larger in the former than in the latter group. Based on the diagnoses registered in the Danish National Psychiatric Register, more patients in the group with symptoms than among patients without symptoms at follow-up were diagnosed with comorbid psychosis although the number was low. Furthermore, based on registrations in the Danish National Psychiatric Register, five patients developed schizophrenia. All of these patients described OC symptoms at followup, and they all had several psychiatric diagnoses. All patients except one continued the use of an antidepressant.

The self-ratings of quality of life were comparable. Both the group with symptoms and the group without symptoms at follow-up reported a significantly higher quality of life during periods without OC symptoms than during periods with OC symptoms (Table 3 ).

The patients were asked to self-rate their personality traits. More patients with OC symptoms than patients without OC symptoms at follow-up described personality traits characterised by anxiety traits $(p=0.03)$. Furthermore, more patients with symptoms described a tendency to schizoid and compulsive traits although the association did not reach significance (Table 3 ).

\section{Predictive factors}

The probability of having OC symptoms at follow-up was higher among patients who had been referred after turning 10 years ( $p=$ 0.028 ) than among patients who had been referred at an earlier age (Table 4). Also, being female increased the risk although this association was not statistically significant ( $p=$ 0.09 ). The occurrence of conduct disorder at baseline increased the risk of long-term OC symptoms; and this association was independent of age at referral and gender $(p<0.001)$. Depression at baseline did not show an association with OC symptoms at follow-up. Having a family member with diagnosed OCD increased the likelihood of OC symptoms at follow-up $(p=0.08)$. This was especially the case, if the family member also reported depressive or anxiety symptoms ( $p=$ 0.038 ) Likewise, a family predisposition to hyperkinetic disorder $(p<0.001)$ and depressive symptoms $(p=0.058)$ entailed an increased risk. The severity of OC symptoms at baseline did not affect the risk of OC symptoms at follow-up. The occurrence of magic obsessions $(p=0.001)$ and repetitive compulsions $(p=0.01)$ increased the risk of OC symptoms at follow-up. Neither avoidance, nor insight, or indecisiveness or responsibility seemed to influence the risk of OC symptoms at follow-up (Table 4).

Twenty-one percent of the group $(\mathrm{N}=20)$ without symptoms at follow-up experienced a late response. Comparing this group with the group of patients having symptoms at followup $(\mathrm{N}=53)$, we found a significant difference in the mean age at referral (group with a late response $12+/-3.1$ years compared with the 
Table 3

Comparing the group of patients without OC symptoms at follow up with the group of patient with continuing OC symptoms.

\begin{tabular}{llll} 
& $\begin{array}{l}\text { No OC symptoms } \\
\text { at follow up } \\
\mathrm{N}=42\end{array}$ & $\begin{array}{l}\text { Continued OC } \\
\text { symptoms } \\
\mathrm{N}=53\end{array}$ & $\begin{array}{l}\text { Statistical } \\
\text { Comparison }\end{array}$ \\
\hline SSRI & 14 & 30 & $p=0.774$ \\
Continued medication & 6 & 28 & $p=0.000$ \\
\hline Comorbidity at follow-up: & 38 & 49 & $p=0.730$ \\
$\quad$ Depressive symptoms & 27 & 44 & $p=0.003$ \\
Eating disorder symptoms & 4 & 13 & $p=0.038$ \\
Tic symptoms & 14 & 16 & $p=0.945$ \\
\hline
\end{tabular}

Comorbidity registered in

the Danish National register:

\begin{tabular}{llll} 
Depression & 5 & 12 & $p=0.175$ \\
Anxiety & 8 & 15 & $p=0.296$ \\
Eating Disorder & 3 & 4 & Too few \\
Tic & 4 & 2 & Too few \\
Psychosis & 0 & 6 & Too few \\
Personality disorder & 1 & 8 & Too few \\
\hline Quality of life - score: & & & \\
In periods with OC symptoms & $8.67+/-6.27$ & $5.82+/-4.18$ & $p=0.000^{*}$ \\
$\quad$ In periods without OC symptoms & $20.0+/-3.62$ & $17.07+/-3.65$ & $p=0.000$ \\
\hline Personality traits - score: & & & \\
Paranoid traits & $5.12+/-3.43$ & $6.65+/-4.02$ & $p=0.13$ \\
Schizoid traits & $2.67+/-3.49$ & $4.32+/-3.5$ & $p=0.08$ \\
Dissocial traits & $2.83+/-3.28$ & $2.59+/-2.42$ & $p=0.74$ \\
Emotional traits & $4.75+/-4.12$ & $6.65+/-4.83$ & $p=0.12$ \\
Compulsive traits & $9.08+/-4.80$ & $11.06+/-4.21$ & $p=0.098$ \\
Anxiety traits & $4.08+/-3.88$ & $6.21+/-3.52$ & $p=0.03$ \\
Dependent traits & $4.38+/-4.18$ & $5.21+/-3.41$ & $p=0.41$ \\
\hline
\end{tabular}

* Quality of life with OC symptoms compared to life without OC symptoms.

group with symptoms at follow-up 15.1+/3.2 years, $p=0.0004)$. Significantly more patients with OCD at follow-up compared with those patients experiencing a late response had relatives with OC symptoms $(p=0.022)$ and depressive symptoms $(p=0.007)$. Fur- thermore, patients who were referred when they were older than 10 years and who had a late response had a significantly lower severity level of baseline OC symptoms $(16.1+/-$ 7.1) than the group with persistent OC symptoms at follow-up $(22.1+/-7.0)(p=0.046)$. 
Table 4

Relative risk of baseline factors on the continuing of OC symptoms seven years after referral.

\begin{tabular}{|c|c|c|}
\hline & Relative risk & Statistical comparison \\
\hline Age of referral $>10$ years & $2.67(1.11-6.40)$ & $p=0.028$ \\
\hline Gender & $1.37(0.95-1.97)$ & $p=0.094$ \\
\hline Comorbidity at baseline & $1.03(0.71-1,51)$ & $p=0.860$ \\
\hline Motor tics & $0.89(0.35-2.25)$ & $p=0.802$ \\
\hline Vocal tic & $1.20(0.45-3.19)$ & $p=0.715$ \\
\hline Depressive symptoms & $1.21(0.78-1.89)$ & $p=0.394$ \\
\hline Eating disorder symptoms & $1.22(0.70-2.12)$ & $p=0.487$ \\
\hline Hyperkinetic disorder & $0.34(0.06-1.97)$ & $p=0.225$ \\
\hline Conduct disorder & $1.81(1.41-2.33)$ & $p=0.000$ \\
\hline \multicolumn{3}{|l|}{ Family disposition to: } \\
\hline OCD diagnosed & $1.57(0.94-2.63)$ & $p=0.084$ \\
\hline Tic diagnosed & $1.08(0.51-2.30)$ & $p=0.834$ \\
\hline Depressive symptoms & $1.86(0.98-3.52)$ & $p=0.058$ \\
\hline Hyperkinetic disorder & $1.93(1.53-2.44)$ & $p=0.000$ \\
\hline Treatment with sertraline at baseline & $0.59(0.38-0.92)$ & $p=0.020$ \\
\hline \multicolumn{3}{|l|}{ Phenotypic presentation: } \\
\hline Obsessions Aggressive & $1.11(0.79-1.57)$ & 0.553 \\
\hline Obsessions Magic & $1.94(1.29-2.92)$ & 0.001 \\
\hline Obsessions Somatic & $0.88(0.63-1.22)$ & 0.445 \\
\hline Obsessions Religious & $0.88(0.64-1.22)$ & 0.441 \\
\hline Obsessions Contamination & $1.08(0.77-1.51)$ & 0.665 \\
\hline Compulsions Checking & $1.29(0.87-1.92)$ & 0.207 \\
\hline Compulsions Repeating & $2.12(1.18-3.81)$ & 0.012 \\
\hline Compulsions Arranging & $1.25(0.86-1.81)$ & 0.235 \\
\hline Compulsions Washing & $0.94(0.68-1.30)$ & 0.712 \\
\hline Compulsions Hoarding & $0.98(0.63-1.54)$ & 0.944 \\
\hline
\end{tabular}

\section{Discussion}

The present study examined a group of children and adolescents 7 years after their referral to a psychiatric hospital for OCD. The study group was defined in a baseline study ${ }^{9}$, and the present study was a clinical prospective study with a response rate of $71 \%$.

At follow-up, 53 participants described OC symptoms, including 2 participants de- 
scribing a relapse of symptoms. Furthermore, 22 participants described remission and 20 participants described a delayed reduction in OC symptoms. Thus, a total of 44 participants $(46 \%)$ showed no symptoms at followup. This distribution is in accordance with the figures reported in a metaanalysis from 2004 (6), which suggested an overall remission rate (not meeting the criteria for full or subthreshold OCD) of $40 \%$. Our findings are also in accordance with the figures reported by Bloch et al. ${ }^{7}$, who found a remission rate of $44 \%$. In our study, a significantly higher proportion of patients with symptoms than of patients without symptoms at follow-up were still receiving medication at the time of the follow-up. This finding corresponds to that of Mikael et al. ${ }^{8}$ who showed that $50 \%$ of the patients continued receiving treatment. Even though the persistence rate of OCD in children and adolescents is lower than the persistence rate in adults, the results of the present study emphasise that OCD may turn into a persistent condition in some younger patients. This is supported by the CYBOCS scores according to which $19.6 \%$ described a worsening of their symptoms, whereas only $43.5 \%$ of the patients experienced a recovery. A significantly larger number of patients with OC symptoms than of patients without OC symptoms at follow-up described an anxious personality trait, and among the former there was a tendency for more patients to describe schizoid and compulsive traits. In a naturalistic 6-month follow-up study, De Haan et $a l .{ }^{15}$ showed that a dimensional score on the PDQ-R for cluster A personality disorder appeared to predict treatment outcome. Cluster A personality disorder includes the odd or eccentric disorders and those who fear social relation, e.g. schizoid personality disorder. In a study from 2011, Ansell et al. ${ }^{16}$ examined the prospective seven year course of several anxiety disorders including OCD. They found that the occurrence of comorbid avoidant personality disorder influenced the course of social phobia and OCD. Furthermore, obsessive compulsive personality disorder was associated with the course of generalised anxiety disorder, OCD and agoraphobia. In the present study, the patients were only asked for traits. However, they experienced traits corresponding to those described in other studies. To our knowledge, the present study is one of the first studies examining personality traits in a follow-up group of children and adolescents with OCD. The participants' quality of life was significantly lower during periods with OCD than during periods without OCD. There was no difference between patients with OC symptoms and remitters. This is in accordance with OCD being on the WHO list of the most burdensome psychiatric disorder in terms of degree of impairment and decreased quality of life OCD ${ }^{4,5}$.

Poor prognostic factors included the phenotypic presentation of magic obsessions and repeating compulsions, a positive family occurrence of OCD, depressive symptoms and hyperkinetic disorder, and referral after the $10^{\text {th }}$ year of age. The occurrence of conduct disorder conferred a negative influence. Bloch et al. ${ }^{7}$ also showed that older age at onset and the occurrence of comorbid ODD were factors heralding a poor prognosis. Others have also shown that parental axis I psychiatric diagnosis ${ }^{5}$ and comorbid psychiatric illness ${ }^{6}$ may affect children and adolescents' prognosis in OCD. A positive family history of either OCD or other psychiatric illnesses has been suggested to be important in relation to the occurrence of OCD in the offspring 5 . A positive family history could influence the family's ability to play a supportive role. In relation to children and young adults, the family is important in retaining the patient in working with the symptoms. The phenotypic presentation of magic obsessions and repeating compulsions as negative prognostic 
factors are in accordance with the findings of Leckman et al. ${ }^{13}$. Leckman et al. ${ }^{13}$ shows that the symmetry/ordering dimension including obsession with symmetry, repeating rituals, counting compulsions and ordering/arranging, often is seen in relation to an early-onset OCD, and that it is associated with concomitant tic disorder, both of which have been associated with a poor outcome ${ }^{17}$. Thus, the findings of the present study may represent the effects of several factors. We were not able to show any importance of a hoarding factor ${ }^{7}$. One explanation may be that the number of children and adolescents with hoarding symptoms was low.

A large number of patients experienced a delayed reduction in their OC symptoms. Compared with the non-responder group, fewer patients in the late responder group reported undergoing combined treatment $(30 \%$ compared with 66\%), and more patients had only psychotherapy (45\% compared with $21 \%$ ). Furthermore, the late responder group had a lower mean age at referral than the non-response group. A family occurrence of depressive symptoms increased the risk of symptoms at follow-up. De Haan et al. ${ }^{15}$ found $38 \%$ to be late responders. They concluded that lack of response after the primary treatment does not necessarily mean a lack of response at follow-up. They suggested that patients with more severe symptoms at baseline could need a longer period of treatment.

Clinical studies may have a disadvantage since the group of patients studied may be very inhomogeneous in terms of both diagnoses and treatment, and it may therefore be difficult to directly compare the effects of treatment. In our study, a proportion of the patients did not want to participate or could not be located. Even though, this group did not differ from the participants in terms of gender, age of referral/age at follow-up, severity of OCD at baseline, treatment or the occurrence of comorbidity and family predisposition, there was a trend towards a lower severity and less comorbidity. Taking together with the fact that the study examines a hospital-based sample, the milder cases of OCD are probably underrepresented.

On the other hand, prospective and observational studies of illness course may have some advantages over follow-up studies of treatment trials. The present prospective study followed patients for seven years, which is one of the longest follow-up studies conducted on children and adolescents with OCD. A clinical study may reflect the natural course of OCD in a group of children and adolescents with OCD including treatment.

The present study showed that, for some patients, OCD may be a persistent disorder demanding close follow-up. The study confirmed several previous findings. In addition, the study has added new knowledge concerning the importance of phenotypic presentation, personality traits and quality of life. Furthermore, the late responder group has been described in relation to the group with persistent symptoms. Knowledge of the different ways of responding to treatment is important to ensure the best and most efficient treatment. As to better describe and characterise possible subgroups of OCD patients abd important predictive factors, future studies should focus on both the knowledge from randomised controlled treatment studies, genetic aspects and neuroimaging studies. An important aspect will be the number of included patients and as such larger scale studies are warranted. 


\section{Acknowledgments and Disclosure of Interest}

The authors wish to thank Erik Parner for valuable statistical advice.

Per Hove Thomsen has received a speaker's fee from Novartis, Medice and Jannsen. All other authors declare that they have no conflicts of interest

\section{References}

1. Blier P, Habib R, Flament MF. Pharmacotherapies in the management of obsessive-compulsive disorder. Can J Psychiatry. 2006; 51 (7): 417-430.

2. Pediatric OCD Treatment Study (POTS) Team. Cognitive-behavior therapy, sertraline, and their combination for children and adolescents with obsessive-compulsive disorder: The Pediatric OCD Treatment Study (POTS) randomized controlled trial. JAMA. 2004; 292 (16): 1969-1976.

3. Garcia AM, Sapyta JJ, Moore PS, Freeman JB, Franklin ME, March JS, et al. Predictors and moderators of treatment outcome in the Pediatric Obsessive Compulsive Treatment Study (POTS I). J Am Acad Child Adolesc Psychiatry. 2010; 49 (10): 1024-1033.

4. Thomsen PH, Mikkelsen HU. Course of obsessivecompulsive disorder in children and adolescents. J Am Acad Adolesc Psychiatry. 1995; 34: 1432-1440.

5. Leonard HL, Swedo SE, Lenane MC, Rettew DC, Hamburger SD, Bartko JJ, et al. A 2- to 7-year follow-up study of 54 obsessive-compulsive children and adolescents. Arch Gen Psychiatry. 1993; 50: 429-439.

6. Stewart SE, Geller DA, Jenike M, Pauls D, Shaw D, Mullin B, et al. Long-term outcome of pediatric obsessivecompulsive disorder: A meta-analysis and qualitative review of the literature. Acta Psychiatr Scand. 2004; 110 (1): 4-13.

7. Bloch MH, Craiglow BG, Landeros-Weisenberger A, Dombrowski PA, Panza KE, Peterson BS et al. Predictors of Early Adult Outcomes in Pediatric-Onset Obsessive-Compulsive Disorder. Pediatrics. 2009; 124: 1085-1094.

8. Micali N, Heyman I, Perez M, Hilton K, Nakatani E, Turner C, et al. Long-term outcomes of obsessive-compul- sive disorder: Follow-up of 142 children and adolescents. $\mathrm{Br}$ J Psychiatry. 2010; 197 (2): 128-134.

9. Nikolajsen KH, Nissen JB, Thomsen PH. Obsessivecompulsive disorder in children and adolescents. Symptom dimensions in a naturalistic setting. Nord J Psychiatry. 2011; 65 (4): 244-250.

10. Goodman WK, Price LH, Rasmussen SA. Children's Yale-Brown Obsessive Compulsive Scale (CY-BOCS). New Haven: Department of Psychiatry, Yale University School of Medicine; 1991.

11. March JS, Biederman J, Wolkow R, Safferman A, Mardekian J, Cook EH, et al. Sertraline in children and adolescents with obsessive-compulsive disorder. JAMA. 1998; 280: 1752-1756.

12. Ruxton GD. The unequal variance t-test is an underused alternative to Student's t-test and the Mann-Whitney U test. Behav Ecol. 2006; 17: 688-690.

13. Leckman JF, Grice DE, Boardman J, Zhang H, Vitale A, Bondi C, et al. Symptoms of obsessive-compulsive disorder. Am J Psychiatry. 1997; 154: 911-917.

14. Peduzzi P, Concato J, Feinstein AR, Holford TR. Importance of events per independent variable in proportional hazards regression analysis. II. Accuracy and precision of regression estimates. J Clin Epidemiol. 1995; 48 (12): 1503-1510.

15. De Haan E, Van Oppen P, van Balkom AJ, Spinhoven P, Hoogduin K, von Dyck R. Prediction of outcome and early $v s$. late improvement in OCD patients treated with cognitive behaviour therapy and pharmacotherapy. Acta Psychiatr Scand. 1997; 96 (5): 354-361.

16. Ansell E, Pinto A, Egan SJ, Edelen M, Markowitz J, Sanislow C, et al. The association of personality disorders with the prospective 7-year course of anxiety disorders. Psychol Med. 2011; 41 (5): 1019-1028.

17. Jakubovski E, Diniz JB, Valerio C, Fossaluza V, Belotto-Silva C, Gorenstein C, et al. Clinical predictors of long-term outcome in obsessive-compulsive disorder. Depress Anxiety. 2013; 30 (8): 763-772.

Corresponding author:

Judith Becker Nissen

Children and Adolescent Psychiatric Centre

Risskov

Skovagervej 2

DK-8240 Risskov

Denmark

0114578473350

E-mail: judiniss@rm.dk 